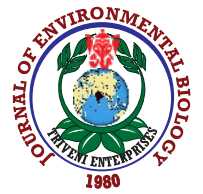

\title{
Lipid and carotenoid production by Rhodosporodium toruloides ATCC 204091 using C5 and C6 sugars obtained from lignocellulosic hydrolysate
}

\author{
S. Sinha, G. Singh and D. Paul ${ }^{\star}$ \\ Amity Institute of Biotechnology, Amity University, Noida- 201313 , India \\ *Corresponding Author Email : dpaul@amity.edu
}

\section{Abstract}

Aim: This study investigated the production of lipids and carotenoids and associated growth properties by the oleaginous red yeast Rhodosporodium toruloides strain ATCC 204091, using C5 and C6 sugar streams of lignocellulosic waste hydrolysate separately and in combination (C5+C6).

Methodology: Cell density, wet and dry biomass weight, concentration of total sugars and reducing sugars were determined at various time intervals during cell growth in media containing $\mathrm{C} 5, \mathrm{C} 6$ and mixed sugars. Lipid and carotenoids were extracted and the media were compared with respect to production.

Results: Production of lipid (22.25\%) and carotenoids $\left(19 \mathrm{mgl}^{-1}\right)$ was highest in C6 sugar, as compared to mixed sugars and C5 sugar.

Interpretation: Due to the versatility of Rhodosporodium toruloides strain ATCC 204091 for utilizing C6 and C5 sugars present in waste hydrolysates, it has been projected as a good choice for cultivation in "waste" hydrolysates.

Key words: Carotenoid, hydrolysate; lignocellulosic, lipid, Rhodosporodium toruloides

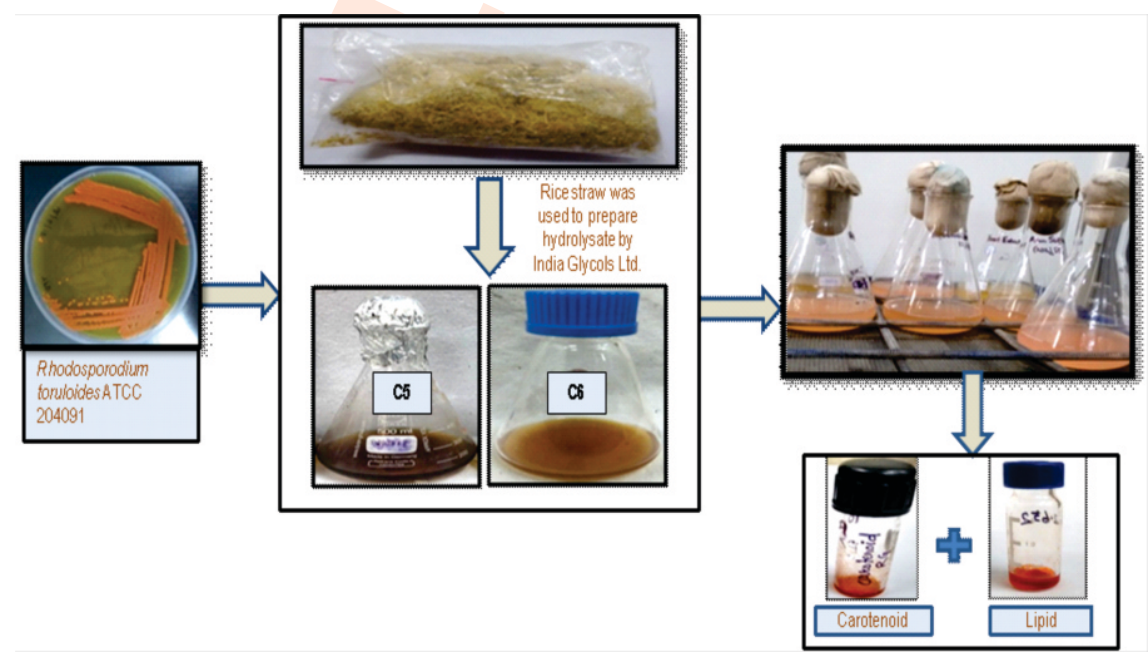

How to cite : Sinha, S., G. Singh and D. Paul: Lipid and carotenoid production by Rhodosporodium toruloides ATCC 204091 using C5 and C6 sugars obtained from lignocellulosic hydrolysate. J. Environ. Biol., 42, 938-944 (2021). 


\section{Introduction}

Growing energy demands worldwide has led to environmental pollution and global warming. The use of fossil fuels has contributed significantly to greenhouse gases (Bos and Hamelinck, 2014), along with the smoke arising due to the burning agricultural waste every year. Agriculture based industries also generate a large amount of residues which are disposed off either by burning, unplanned land filling or by dumping on unused land (Sadh et al., 2018). Concerns over environmental deterioration as well as rising energy prices in recent years has led to evolution of alternative environmentfriendly initiatives and renewable bioenergy resources (Okonko et al., 2009). Growing population and rapid development in industrialization in most of the developing countries demand sustainable generation of economical energy resources, e.g., using inexpensive agricultural wastes. Agro-industrial lignocellulosic waste is being widely studied for their application in producing antioxidants, antibiotics, animal feed, biofuels, enzymes and vitamins by culturing a variety of microorganisms on waste hydrolysate (Ravindran and Jaiswal, 2016; Slininger et al., 2016; Sadh et al., 2018; Tsegaye et al., 2019).

Oleaginous yeasts can accumulate lipids upto $20 \%-85 \%$ as storage compound of the biomass and can be harnessed for sustainable lipid production (Kosa et al., 2017). Several studies have demonstrated that Rhodosporidium sp. of yeast can accumulate upto $70 \%$ of its biomass as lipids and is highly adaptable to several types of substrates (Singh et al., 2018a; Osorio-González et al., 2019a). Therefore, such oleaginous yeast have the ability to grow in varied sugar content media, to produce various value added, products that are considered to be industrially useful (Mezzomo and Ferreira, 2016). A variety of inexpensive substrates like agro-industrial waste (Li et al., 2008), food waste /municipal wastewater have been used to produce alternate energy (Liang et al., 2006; Sales-Campos et al., 2013) and have now been considered as a valuable feedstock. Inspite of using, inexpensive carbon source as substrate, and the microbial biodiesel is not able to fight with the conventional petroleum diesel on a commercial scale, because of high expenses of lipid production, extraction and conversion. In order to reach the economic feasibility of microbial biodiesel production, the marketing of higher valued products that are formed in the course of microbial fermentation of feedstock is another option. Depending on the substrate and microorganism used, this can be met by concomitantly producing, speciali chemicals, biosurfactants or certain pigments (Pterik et al., 2013; Singh et al., 2016; Kot et al., 2018; Singh etal., 2018 b).

The economic restrictions concerning the application of microbial oils as renewable resource for biodiesel production can be overcomed in this way and cultivation of oleaginous yeast is, therefore, the focus of research. Red yeast $R$. toruloides has the ablility to produce certain carotenoids, namely $\beta$-carotene, torulene and torularhodin (important antioxidants and provitamins) in the course of microbial fermentation (Buzzini et al., 2007; Kot et al., 2018). The major sugars generated by the hydrolysis of lignocellulosic biomass from agro industrial waste are glucose and xylose. The purification process of sugars from hydrolysate is a costly affair, therefore, the crude hydrolysate should be utilized for substrate forfermentation. $R$. toruloides has been studied as a model organism for lipid production and has shown to co-utilize both hexose and pentose sugars (Wiebe et al., 2012). The present study focus on the production of lipid and carotenoid from the yeast $R$. toruloides using lignocellulosic sugar hydrolysate (containing both hexoses and pentoses) generated from rice-straw. Therefore, the proposed bioprocess aims at solving environmental issues by utilizing waste as growth media on one hand, and on the other hand beat the financial restrictions for biodiesel production via cost-effective strategy. The aim of this study was to investigate the potential of oleaginous red yeast strain $R$. toruloides to convert the lignocellulosic waste hydrolysate to valuable lipids and carotenoids by comparing the usage of all carbons sources present in the hydrolysate.

\section{Materials and Methods}

Microorganism and culture media: The yeast $R$. toruloides ATCC204091 culture was obtained from the College of Veterinary Medicine Mississippi State University, USA. The yeast was maintained on Yeast extract, Peptone, Dextrose (YPD) agar plates and stored at $4^{\circ} \mathrm{C}$ for further use and sub cultured at regular intervals. Minimal Medium was used for growth of inoculum. Lignocellulosic rice straw hydrolysate was obtained from India Glycols Pvt Ltd., in the form of condensate. It was further used as growth medium. The growth medium contained specified carbon source glucose $\left(28 \mathrm{~g} \mathrm{l}^{-1}\right)$, xylose $\left(24 \mathrm{~g} \mathrm{l}^{-1}\right)$ and combination of glucose and xylose $\left(28 \mathrm{~g} \mathrm{l}^{-1}\right)$. The $\mathrm{pH}$ of the medium was maintained at 5.5. In the first stage of inoculum preparation, the yeast cells were inoculated into a liquid medium of a composition identical to one to be used in a given fermentation. This was called seed culture. The liquid seed culture was incubated at room temperature for $48 \mathrm{hr}$. The resulting culture was used as inoculum for all batch fermentation experiments. The inoculum size used was $10 \%$ in the final fermentation medium. The chemicals and reagents used were of analytical grade.

Culture conditions: An inoculum with optical density 0.7 to 0.8 at $600 \mathrm{~nm}$ was used to inoculate the flask containing $50 \mathrm{ml}$ of fermentation medium. These flasks were kept on a shaker at 120 rpm for $136 \mathrm{hr}$.

Fermentations using hydrolysates of $\mathbf{C} 5$ and $\mathbf{C} 6$ sugars as carbon source: The fermentation medium was prepared using all the specified components of minimal medium, except glucose. Instead of pure glucose, C5 and C6 sugars from the hydrolysate were added to the medium. A $50 \mathrm{ml}$ of fermentation medium was carried in $250 \mathrm{ml}$ Erlenmeyer flasks and the flasks were held at room temperature on shaker at $120 \mathrm{rpm}$.

Analytical method: Dry cell weight was calculated after centrifuging $1 \mathrm{ml}$ of fermentation medium in pre-weighed vials at 
$4000 \mathrm{rpm}$ for $5 \mathrm{~min}$. The supernatant was used to determine total and reducing sugars and cell pellets were weighed and dried in an oven at $80^{\circ} \mathrm{C}$ for dry cell weight. To determine glucose in the supernatants that used hydrolysate of C6 industrial sugar as sole carbon source, nitro salicylic acid method (Miller, 1959) was followed. Same method was applied to measure reducing sugar in the hydrolysate of $\mathrm{C} 5$ industrial sugar. The absorbance was read at $540 \mathrm{~nm}$ in both case. The standard was D-glucose and D-xylose, depending on the carbon source used in the culture medium. Phenol sulphuric acid method was followed to determine the total sugar of the medium at $490 \mathrm{~nm}$ absorbance (Dubois, 1956).

HPLC analysis: HPLC analysis was performed for the detection of different sugars in the lignocellulosic hydrolysate stream C5 and $\mathrm{C} 6$, using $5 \mathrm{mM} \mathrm{H}_{2} \mathrm{SO}_{4}$ in MilliQ water as mobile phase in a HPLC column Aminex HPX-87P, 300 X 7.8 mm (Bio-Rad, India). The flow rate was $0.5 \mathrm{~m} \mathrm{~min}^{-1}$. Occurrence of different sugars and their concentration in the hydrolysate was determined by HPLC analysis.

Lipid extraction: Lipid extraction was done by Bligh and Dyer (1959) method after some modifications. The mixture of chloroform and methanol, (2:1) was added to dry biomass in a test tube. The total solvent system had $2 \mathrm{ml}$ chloroform and $1 \mathrm{ml}$ methanol for each gram of biomass. After adding chloroform and methanol to the cells, the mixture was allowed to vortex for $15 \mathrm{~min}$ followed by adding $1 \mathrm{M} \mathrm{NaCl}$. This combination was centrifuged for $15 \mathrm{~min}$ at $3000 \mathrm{rpm}$ to split its aqueous and organic phase. The lower phase was transferred to pre weighed vial and dried until its organic phase was evaporated. Lipids were measured in triplicates and the average value was reported.

Carotenoid extraction: Carotenoids was extracted using the method of Hiscox and Israelstam (1979). Cells were collected by centrifugation and treated with Dimethyl sulfoxide at $50^{\circ} \mathrm{C}$ for $1 \mathrm{hr}$. This process was repeated till the pellets turned colourless and the entire carotenoids were transferred in DMSO. The supernatant afterward transferred to hexane and the upper layer was separated by liquid-liquid extraction. The optical density was determined spectrophotometerically by Systronics UV-Vis spectrophotometer at $450 \mathrm{~nm}$ against hexane. Quantification was made via standard curve of $\beta$-carotene as reported earlier.

Statistical analysis: All experiments were performed in triplicates. All statistical calculations were done using MEDCALC Software bvba statistical software (www.medcalc.org).

\section{Results and Discussion}

The inoculum size of $10 \%$ by volume resulted in higher biomass production, compared to smaller inocula when grown in Minimal Medium. Chisti and Young (1999) stated that a small inoculum generally have a long lag phase and an extended duration of fermentation as compared to large inoculum for an industrial bioreactor. Based on this study, further work has been done using a $10 \%$ by volume inoculums. $R$. toruloides is known for high lipid production with additional significant chemicals because it can be easily cultivated on glucose, xylose and other sugars present in hydrolysates of waste biomass as well as lignocelluloses (Wiebe et al., 2012; Bommareddy et al., 2015). To improve the quality with decreased expenditure of microbial oil production, cheap locally available media should be used to replace synthetic fermentation media (Singh et al., 2020).

Different oil producing yeast have been grown on cheap media to generate lipids, like Yarrowia lipolytica was cultivated in molasses (waste generated by sugarcane industry), oleaginous filamentous fungus Aspergillus oryzae in potato processing wastewater and Cunninghamella echinulata on the media prepared by tomato waste hydrolysate (Muniraj et al., 2013; Rakicka et al., 2015; Madonna et al., 2016). Inspite of lipid production, there are many carotenogenic yeasts which can incorporate various carbon sources such as glucose, xylose, cellobiose, sucrose, glycerol, sorbitol, etc., and accordingly a variety of waste materials can be used as inexpensive substrates for their cultivation (Petrik et al., 2013). Microorganisms are normally capable to metabolize glucose easily, hence, the fermentation was first carried out with the hydrolysate of C6 sugar as sole carbon source. HPLC analysis revealed that the composition of industrial lignocellulosic hydrolysate streams had glucose $\left(26 \mathrm{gl}^{-1}\right)$ and xylose $\left(2 \mathrm{gl}^{-1}\right)$. The concentration of glucose was initially $26 \mathrm{~g} \mathrm{I}^{-1}$, and it was consumed within $112 \mathrm{hr}$ of fermentation with higher growth rate and a higher final biomass concentration (13.25 g l-1, Fig. 1 a). Since it was crude hydrolysate and contained a mixture of sugars, the fermentation was carried out for $136 \mathrm{hr}$, for consumption of all the sugars present in it. The biomass yield on C6 sugar was 0.392. C5 is a monosaccharide with five carbon atom. Several microorganisms are normally not able to utilize C5 sugars (Saccharomyces cerevisiae), while some others (Pichia stipitis) metabolize it efficiently (Thanapimmetha et al., 2019). R. toruloides yeast has the ability to metabolize glucose as well as xylose.

The potential of $R$. toruloides to use xylose was assessed when it was grown in the media having pure xylose C5 sugar as the sole carbon source (Singh et al., 2018b). The industrial lignocellulosic C5 hydrolysate stream having total sugars of $24 \mathrm{gl}^{-}$ ${ }^{1}$, comprised of xylose $\left(16 \mathrm{gl}^{-1}\right)$, glucose $\left(5 \mathrm{gl}^{-1}\right)$ and arabinose $(3 \mathrm{~g}$ $\left.I^{-1}\right)$ as analyzed by HPLC. $R$. toruloides yeast strain was able to grow on xylose, though its growth was very slow exhibiting distinct lag phase (0-32 hr, Fig 1b). The cells were harvested from the xylose rich medium after $136 \mathrm{hr}$ of cultivation and the final biomass was $6.5 \mathrm{~g} \mathrm{l}^{-1}$. The biomass yield on C5 sugar was 0.270 . This medium comprised of both $\mathrm{C} 5$ and $\mathrm{C} 6$ mixed sugars. The concentration of mixed sugars was $28 \mathrm{gl}^{-1}$ in the medium. Glucose was consumed before xylose during fermentation as observed during its growth on "mandi" waste hydrolysate (Singh et al., 2018b; Sinha et al., 2020). Almost all glucose was consumed within $80 \mathrm{hr}$, however, the fermentation was carried up to $136 \mathrm{hr}$ where the residual sugar was $1.4 \mathrm{gl}^{-1}$ (Fig. 1c). The concentration of biomass was $8 \mathrm{gl}^{-1}$ at $136 \mathrm{hr}$. Biomass yield was 0.285 . This result was almost similar to the biomass yield on C5 sugar (= 

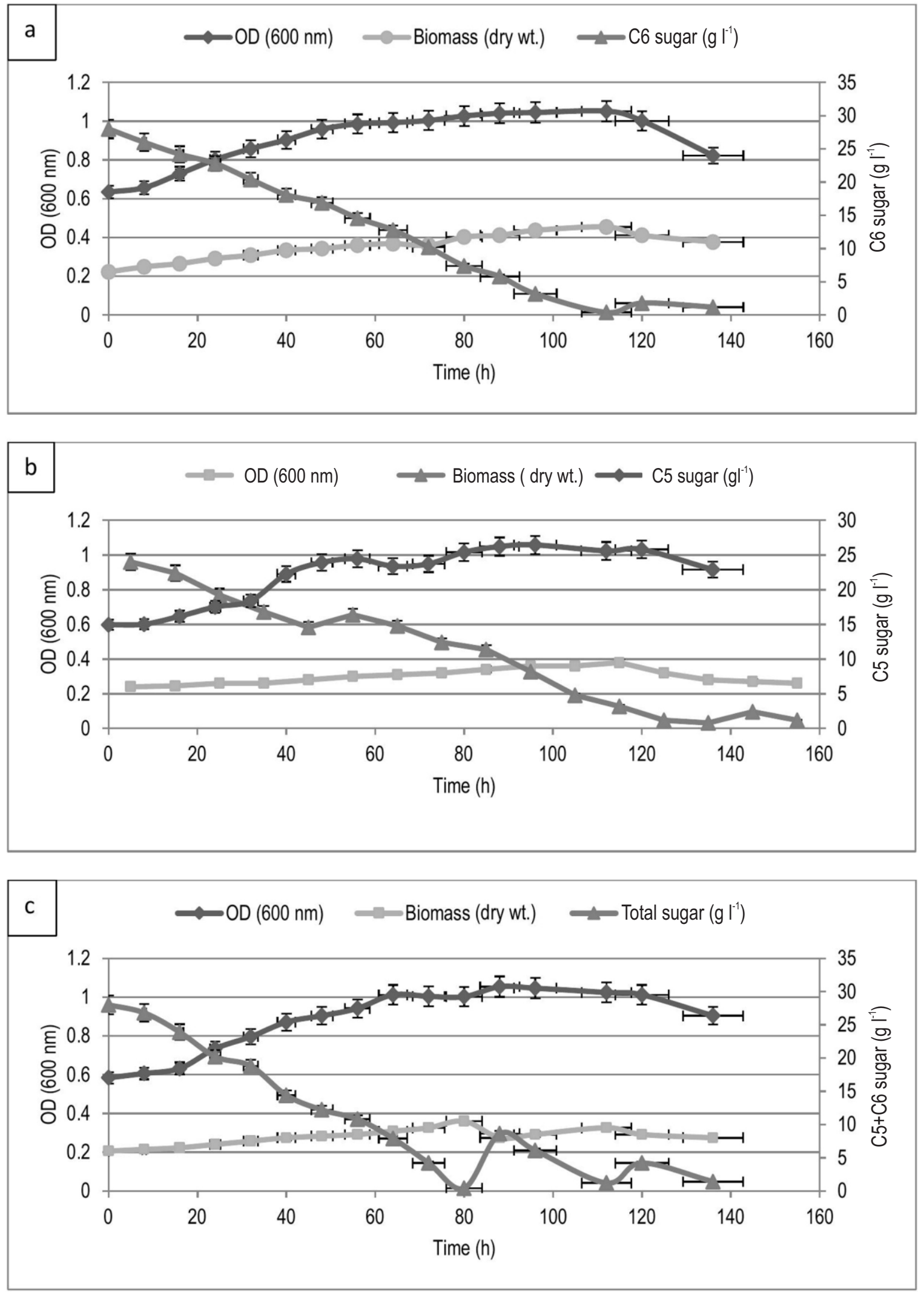

Fig. 1: Study of growth, biomass production and sugar utilization by R. toruloides in (a) C6, (b) C5 and (c) C6+C5 sugar streams of lignocellulosic hydrolysate. All the experiments were performed in triplicates, and the standard deviation was reported with error bars. Differences were measured significant at $p<0.05$ level. 


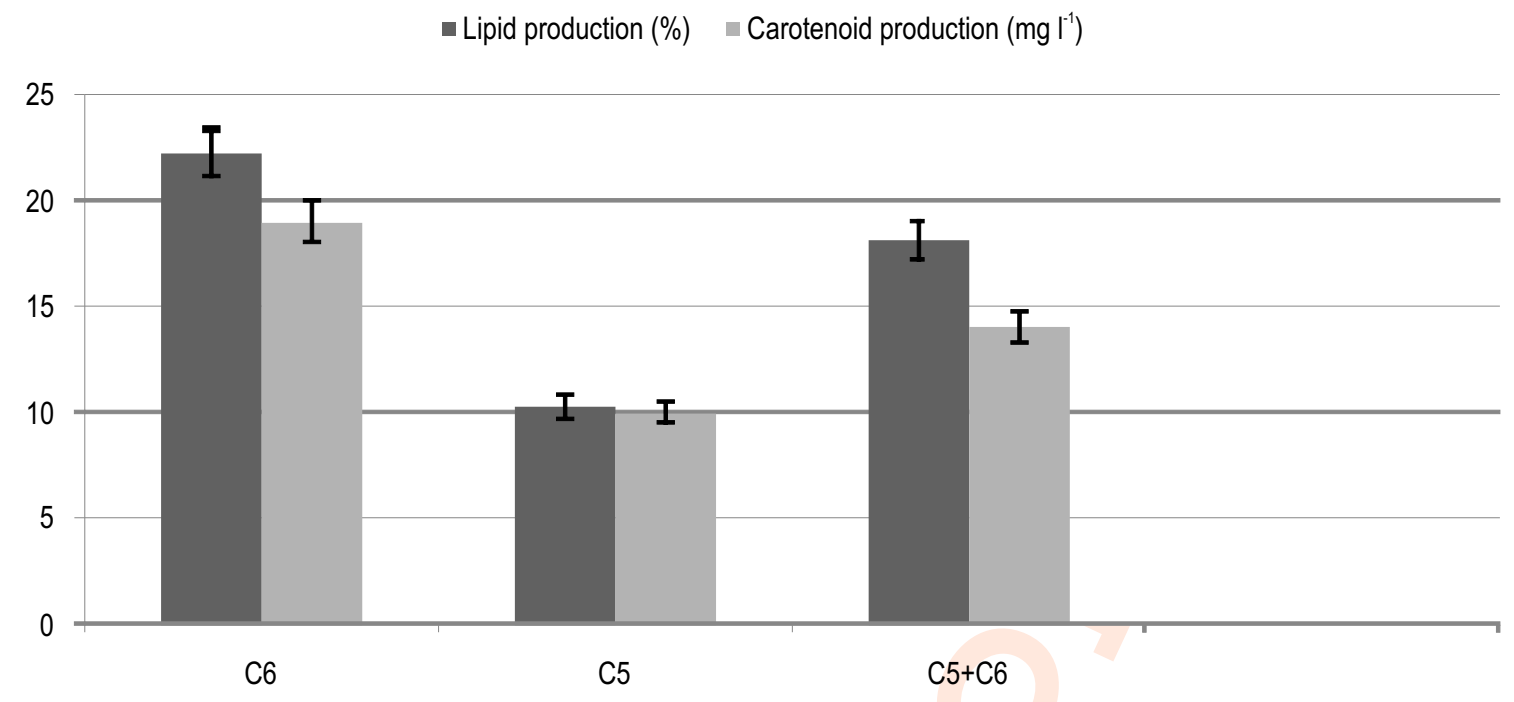

Fig. 2: Lipid production and carotenoid production $\left(\mathrm{mgl}^{-1}\right)$ by $R$. toruloides using $\mathrm{C} 6, \mathrm{C} 5$ and $\mathrm{C} 6+\mathrm{C} 5$ sugar streams of lignocellulosic hydrolysate.

$0.270)$, but $27.2 \%$ less than the yield on C6 sugar (=0.392). The oleaginous yeast $R$. toruloides was able to grow and accumulate lipids as well as produce carotenoids in media containing $\mathrm{C} 6$ sugar as the sole carbon source. Various filamentous fungi and yeast acquire higher concentrations of lipids in their biomass. The genus Rhodosporidium of yeast was used as model organism for the production of lipids and carotenoids because their strains have outstanding capabilities of concomitant production of lipid and carotenoids and wide adaptabilities to a variety of substrates (Xu and Liu, 2017; Singh et al., 2018b). Generally, the most supplied carbohydrate is glucose which has been studied for the production of lipids in yeast (Chetna et al., 2015). Hydrolysates (Tsigie et al., 2011) on the mixed sugars and waste water (Muniraj et al., 2013) are now rapidly being used for the production of lipid directly.

High cell density cultures are preferred for industrial production of lipids, but the oxygen demand for the process limits the density of biomass obtained in flask cultures at small scale. Osorio-Gonzalez et al. (2019b) showed that $R$. toruloides-1588 strain produced highest biomass yield corresponding to the maximum lipid yields ( $0.35 \mathrm{~g}$ lipids $\mathrm{g}^{-1}$ biomass) at $112 \mathrm{hr}$ of fermentation in C-6 hydrolysate culture media. In this study, the highest lipid (22.25\%) and carotenoid content ( $\mathrm{m} \mathrm{g} \mathrm{l}^{-1}$ of dry cell weight) produced by $R$. toruloides was found after $112 \mathrm{hr}$ of cultivation in $\mathrm{C} 6$ sugar (Fig. 2). R. toruloides strain can metabolize a variety of substrates (Papanikolaou and Aggelis, 2011), as well as non-detoxified lignocellulosic hydrolysates (Bonturi et al., 2017).

Hemicellulosic hydrolysates and raw glycerol are potential inexpensive substrates for microbial production of lipids (Lopes et al., 2020; Chmielarz et al., 2021). Hemicellulose is the second most plentiful fraction of lignocellulose and its extraction cum hydrolysis produces xylose as a main component (along with other C5 and some C6 sugars) besides acetic acid (Chandel et al., 2013). Xylose is a five-carbon atom (C5) monosaccharide which is the second most plentiful sugar present in plant biomass (Tiukova et al., 2019). Its consumption by microorganisms is considered as a challenge to attain a sustainable valorization of plant biomass and commercialization of lignocellulosic fuels and chemicals (Nogué and Karhumaa, 2015; Zha et al., 2021). The yeast was able to produce lipid and carotenoids simultaneously when grown in xylose-rich medium of hydrolysate (C5), but the final biomass and lipid production was lesser than that of glucose rich medium (C6). The highest lipid content in the biomass recovered from C5 sugar was $10.25 \%$ whereas the carotenoid content was $m$ $\mathrm{gl}^{-1}$ of dry cell weight in the biomass harvested after fermentation (Fig. 2). R. toruloides competently utilize different carbon sources, like arabinose, xylohexose, xylitol, succinate acid etc., with glucose, xylose and glycerol, which are prime components for growth and lipid formation (Singh et al., 2018b). In this study, the microorganism efficiently grew in the media comprising C5 and C6 sugar as mixed carbon sources to for the production of lipids and carotenoids.

The concentration of final biomass recovered after harvesting was $8 \mathrm{gl}^{-1}$ (Fig. $1 \mathrm{c}$ ). The total lipid content of the final biomass was $18.12 \%$ and the carotenoid content was $\mathrm{g}^{-1}$ of dry cell weight (Fig. 2). It has was observed that as soon as all the sugars were consumed, the lipid content of the biomass reduced. Hence, a vigilant timing of harvesting is important to make sure that sugars are totally utilized and the biomass is harvested when the lipid content is at its climax. It may be therefore accepted that Rhodosporidium toruloides is emerging as a promising strain for the conversion of lignocellulosic biomass into valuable lipids and carotenoids because of its versatile ability of utilizing various carbon sources. This report provides a proper bioprocess for the production of lipid and carotenoids, using C5 and C6 sugar streams of lignocellulosic waste hydrolysate as low cost substrate. The study meant at improvement of environmental 
concerns about safe disposal of such waste, along with the production of invaluable carotenoids and microbial lipid, which can be further used for the production of biodiesel via sustainable and cost-effective approach which may be scaled up in future.

\section{Acknowledgments}

The authors acknowledge the constant support and appreciation of our respected Founder President, Amity University and the Director, AIB, AUUP. We also acknowledge Sr. S.R Soni (Adjunct Professor, AIB) and Mr. Umesh Joshi, Senior Manager of India Glycols Ltd. (IGL), Kashipur, Uttarakhand, India, for providing lignocellulosic hydrolysates.

\section{Add-on Information}

Authors' contribution: S. Sinha: Conceptualization, Methodology, Investigation, Writing original draft, Experimentation and Data interpretation; G. Singh: Experimentation and Data interpretation; D. Paul: Supervision, Visualization, Writing, \& editing.

\section{Research content: The research content of manuscript is original} and has not been published elsewhere.

\section{Ethical approval: Notapplicable}

Conflict of interest: The authors declare that there is no conflict of interest.

\section{Data from other sources: Not applicable}

Consent to publish: All authors agree to publish the paper in Journal of Environmental Biology.

\section{References}

Bligh, E.G. and W.J. Dyer: A rapid method of total lipid extraction and purifcation. Can. J. Biochem. Physiol., 37, 911-917 (1959).

Bommareddy, R.R., W. Sabra, G. Maheshwari and A.P. Zeng: Metabolic network analysis and experimental study of lipid production in Rhodosporidium toruloides grown on single and mixed substrates. Microb. Cell. Factories., 14, 36 (2015).

Bonturi, N., A. Crucello, A.J.C. Viana and E.A. Miranda: Microbial oil production in sugarcane bagasse hemicellulosic hydrolysate without nutrient supplementation by a Rhodosporidium toruloides adapted strain. Proc. Biochem., 57, 16-25 (2017).

Bos, A. and C. Hamelinck: Greenhouse gas impact of marginal fossil fuel use. Project number: BIENL14773. (2014)

Buzzini, P., M. Innocenti, B. Turchetti, D. Libkind, M. V. Brook and N. Mulinacci: Carotenoid profiles of yeast belonging to the genera Rhodotorula, Rhodosporidium, Sporobolomyces and Sporidiobolus. Can. J. Microbiol., 53, 1024-1031 (2007).

Chandel, A.K., S. Silvério and O.V. Singh: Detoxification of lignocellulose hydrolysates: Biochemical and metabolic engineering toward white biotechnology. BioEnergy Res., 6, 388-401 (2013).

Chetna, S.M., M.K. Nagra, Gunjan, A. Khurana, P. Sharma, K.K. Bandyopadhyay and D. Paul: Cultivation of oleaginous yeast on extracts on inedible parts of vegetables, fruits for lipid production. Int. J. Bas. Appl. Biol., 2, 329-332 (2015).

Chisti, Y. and M. Moo-Young: Fermentation technology, bioprocessing, scale-up and manufacture. Biotechnol.-The Science and the Business, pp. 167-209 (1991)

Chmielarz, M., J. Blomqvist, S. Sampels, M. Sandgren and V. Passoth: Microbial lipid production from crude glycerol and hemicellulosic hydrolysate with oleaginous yeasts. Biotechnol. Biofuels, 14, 1-11 (2021).

Dubois, M., K.A. Gilles, J.K. Hamilton, P.A. Rebers and F. Smith: Colorimetric method for determination of sugars and related substances. Anal. Chem., 28, 350-356 (1956).

Hiscox, J.D. and G.F. Israelstam: A method for the extraction of chlorophyll from leaf tissue without maceration. Can. J. Bot., 57, 1332-1334. (1979).

Kosa, G., A. Kohler, V. Tafintseva, B. Zimmermann, K. Forfang, N.K Afseth, D. Tzimorotas, K.S. Vuoristo, S.J. Horn, J. Mounier and V. Shapaval: Microtiter plate cultivation of oleaginous fungi and monitoring of lipogenesis by high-throughput FTIR spectroscopy. Microb. Cell Fact., 16, 1-12 (2017).

Kot, A.M., S. Błażejak, I. Gientka, M. Kieliszek and J. Bryś: Torulene and torularhodin: "new" fungal carotenoids for industry?. Microb. Cell Fact., 17, 49 (2018).

Li, Q., W. Du and D. Liu: Perspectives of microbial oils for biodiesel production. Appl. Microbiol. Biotechnol., 80, 749-756 (2008).

Liang, X.A., W.B. Dong, X.J. Miao and C.J. Dai: Production technology and influencing factors of microorganism grease. Food. Res. Dev., 27, 46-47 (2006)

Lopes, H.J.S., N. Bonturi, E.J. Kerkhoven, E.A. Miranda and P.J. Lahtvee: $\mathrm{C} / \mathrm{N}$ ratio and carbon source-dependent lipid production profiling in Rhodotorula toruloides. Appl. Microbial. Biotechnol., 104, 2639-2649 (2020)

Madonna, S.S., J. Ranjitha and S. Vijayalakshmi: Utilization of agro waste as carbon sources for high lipid production by Aspergillus Niger. Int. Chem. Tech. 3, 635-639 (2016).

Mezzomo, N. and S.R. Ferreira: Carotenoids functionality, sources, and processing by supercritical technology: A review. J. Chem., 7, 1-16 (2016).

Miller, G.L.: Use of dinitrosalicylic acid reagent for determination of reducing sugar. Analyt. Chem., 31, 426-428 (1959).

Muniraj, I.K., L. Xiao, Z. Hu, X. Zhan and J. Shi: Microbial lipid production from potato processing wastewater using oleaginous filamentous fungi Aspergillus oryzae. Water. Res., 47, 3477-3483 (2013).

Nogué, V.S. and K. Karhumaa: Xylose fermentation as a challenge for commercialization of lignocellulosic fuels and chemicals. Biotechnol. Lett., 37, 761-772(2015).

Okonko, I.O., O.T. Adeola, F.E. Aloysius, A.O. Damilola and O.A. Adewale: Utilization of food wastes for sustainable development. Elec. J. Env. Agricult. Food Chem., 8, 263-286 (2009).

Osorio-González, C.S., K. Hegde, S.K. Brar, A Kermanshahipour and A. Avalos-Ramírez: Challenges in lipid production from lignocellulosic biomass using Rhodosporidium sp. A look at the role of lignocellulosic inhibitors. Biofuel. Bioprod. Bior., 13, 740 759 (2019a).

Osorio-González, C.S., K. Hegde, P. Ferreira, S.K. Brar,A. Kermanshahipour, C.R. Soccol and A. Avalos-Ramírez: Lipid production in Rhodosporidium toruloides using C-6 and C-5 wood hydrolysate: A comparative study. Biomass Bioenerg., 130, 105355(2019b).

Papanikolaou, S. and G. Aggelis: Lipids of oleaginous yeasts. Part II: Technology and potential applications. Eur. J. Lipid Sci. Technol., 113, 1052-1073 (2011).

Perera, F: Pollution from fossil-fuel combustion is the leading 
environmental threat to global pediatric health and equity: solutions exist. Int. J. Environ. Res. Public HIth., 15, 16 (2018).

Petrik, S., I. Marova, A. Haronikova, I. Kostovova and E. Breierova: Production of biomass, carotenoid and other lipid metabolites by several red yeast strains cultivated on waste glycerol from biofuel production-a comparative screening study. Ann. Microbial., 63, 1537-1551 (2013).

Rakicka, M., Z. Lazar, T. Dulermo, P. Fickers and J.M Nicaud: Lipid production by the oleaginous yeast Yarrowia lipolytica using industrial by-products under different culture conditions. Biotechnol. Biofuels, 8, 104 (2015).

Ravindran, R. and A.K. Jaiswal: Microbial enzyme production using lignocellulosic food industry wastes as feedstock: A review. Bioengineering, 3, 30 (2016).

Sadh, P.K., S. Duhan and J.S. Duhan: Agro-industrial wastes and their utilization using solid state fermentation: A review. Bioresour. Bioprocess., 5, 1 (2018).

Sales-Campos, H., P.R. Souza, B.C. Peghini, J.S. Da Silva and C.R. Cardoso: An overview of the modulatory effects of oleic acid in health and disease. Mini. Rev. Med. Chem., 13, 201-210 (2013).

Singh, G., A. Jawed, D. Paul, K.K. Bandyopadhyay, A. Kumari and S. Haque: Concomitant production of lipids and carotenoids in Rhodosporidium toruloides under osmotic stress using response surface methodology. Front. Microbiol., 7, 1686 (2016).

Singh, G., C. Jeyaseelan, K.K. Bandyopadhyay and D. Paul: Comparative analysis of biodiesel produced by acidic transesterification of lipid extracted from oleaginous yeast Rhodosporidium toruloides. 3 Biotech, 8, 434 (2018a).

Singh, G., S. Sinha, K.K. Bandyopadhyay, M. Lawrence and D. Paul: Triauxic growth of an oleaginous red yeast Rhodosporidium toruloides on waste 'extract' for enhanced and concomitant lipid and $\beta$-carotene production. Microb. Cell Fact., 17, 182 (2018 b).

Singh, G., S. Sinha, K.K. Kumar, N.A Gaur, K.K Bandyopadhyay and D. Paul: High density cultivation of oleaginous yeast isolates in 'mandi'waste for enhanced lipid production using sugarcane molasses as feed. Fuel, 276, 118073 (2020).

Sinha, S, G. Singh, A. Arora and D. Paul: Carotenoid production by red yeast isolates grown in Agricultural and" Mandi" waste. Waste Biomass Valori., 1-11 (2020). DOI https://doi.org/10.1007/ s12649020-01288-8

Slininger, P.J., B.S. Dien, C.P. Kurtzman, B.R. Moser, E.L Bakota, S.R. Thompson, P.J. O'Bryan, M.A. Cotta, V. Balan, M. Jin and L.D.C. Sousa: Comparative lipid production by oleaginous yeasts in hydrolyzates of lignocellulosic biomass and process strategy for high titers. Biotechnol Bioeng., 113, 1676-1690 (2016).

Thanapimmetha, A., N. Peawsuphon, Y. Chisti, M. Saisriyoot and P. Srinophakun: Lipid production by the yeast Lipomyces starkeyi grown on sugars and oil palm empty fruit bunch hydrolysate. Biomass Convers. Bior., 1-14. (2019). . https://doi.org/10.1007/ s13399-019-00532-z

Tiukova, I.A., J. Brandenburg, J. Blomqvist, S. Sampels, N. Mikkelsen, M. Skaugen, M. $\varnothing$ Arntzen, J. Nielsen, M. Sandgren and E.J. Kerkhoven: Proteome analysis of xylose metabolism in Rhodotorula toruloides during lipid production. Biotechnol. Biofuels, 12, 137 (2019).

Tsegaye B., C. Balomajumder and P. Roy: Microbial delignification and hydrolysis of lignocellulosic biomass to enhance biofuel production: an overview and future prospect. Bull. Nati. Res. Centre, 43, 51 (2019).

Tsigie, Y.A., C.Y. Wang, C.T. Truong and Y.H. Ju: Lipid production from Yarrowia lipolytica Po1g grown in sugarcane bagasse hydrolysate. Bioresour. Technol., 102, 9216-9222 (2011).

Wiebe, M.G., K. Koivuranta, M. Penttila and L. Ruohonen: Lipid production in batch and fed-batch cultures of Rhodosporidium toruloides from 5 and 6 carbon carbohydrates. BMC Biotechnol., 12, 26 (2012).

$\mathrm{Xu}, \mathrm{J}$. and D. Liu: Exploitation of genus Rhodosporidium for microbial lipid production. World J. Microbiol. Biotechnol., 33, 54 (2017).

Zha, J., M., Yuwen, W. Qian, and X Wu: Yeast-based biosynthesis of natural products from xylose. Front. Bioeng. Biotechnol., 9, 26 (2021). 